Supporting Information

\title{
Fluorocarbon Rubber-based Inert Dry Adhesive for Applications in Harsh Conditions
}

Sung Ho Lee ${ }^{1, t}$, Hyo Sung Kim ${ }^{2, t}$, Hyun Woo Song ${ }^{2}$, Moon Kyu Kwak ${ }^{2, *}$

1. Electrical Electronics and Computer Science, University of Michigan, Ann Arbor, MI 48105,

USA

2. Department of Mechanical Engineering, Kyungpook National University, Daegu 41566,

Republic of Korea

‡ These authors contributed equally to this work.

* Corresponding author:mkkwak@knu.ac.kr 
(a)

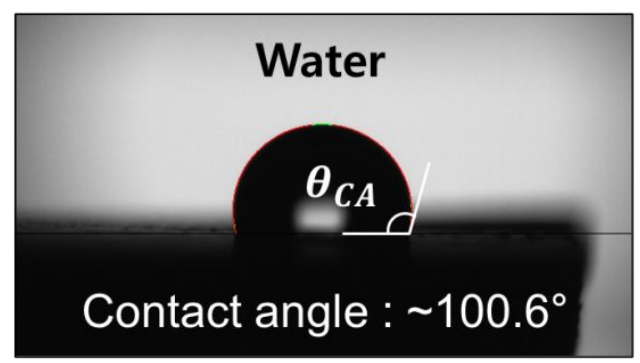

(b)

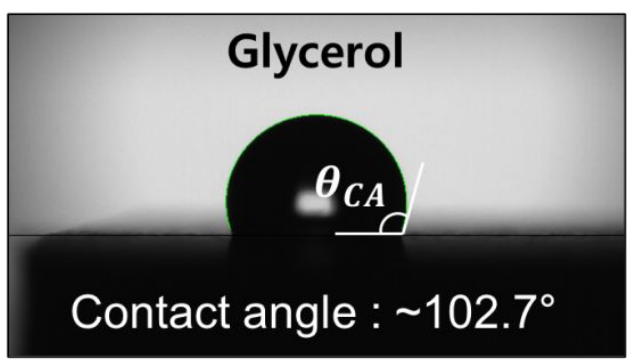

Figure S1. Measurement of contact angle by using water and glycerol.

(a)

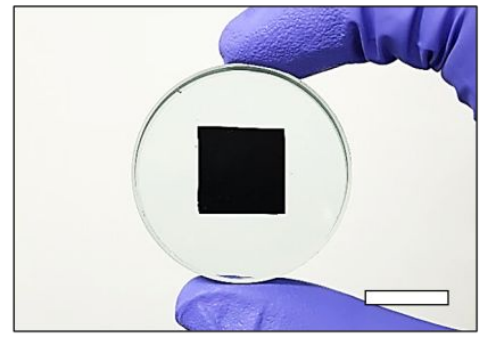

(c)

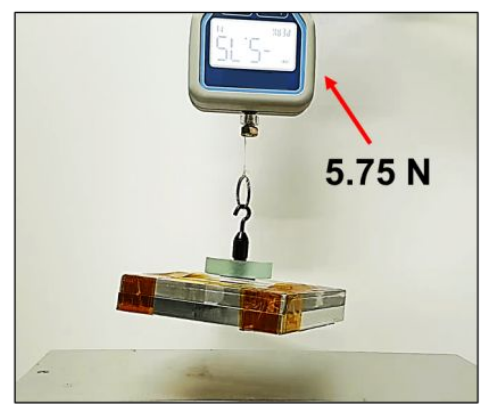

(b)

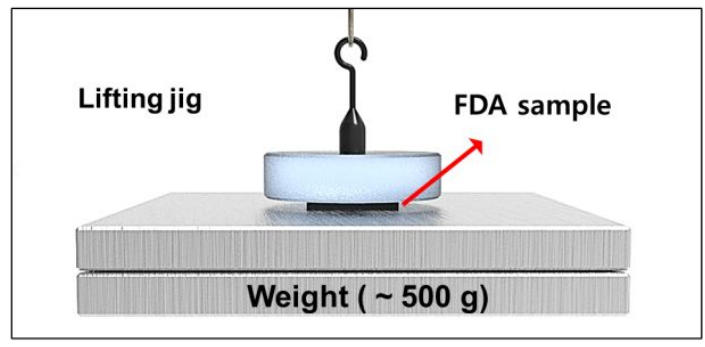

(d)

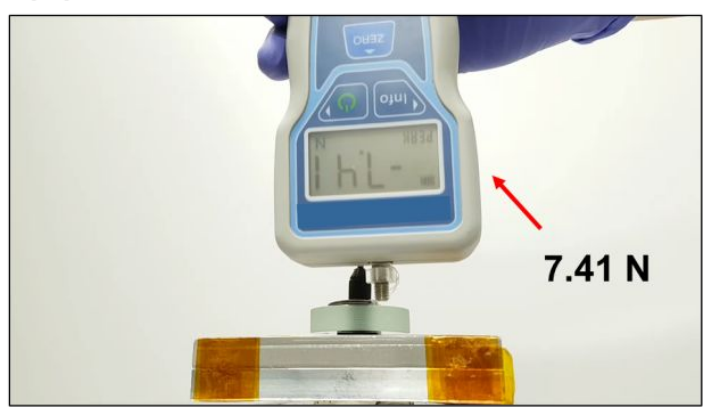

Figure S2. (a) Photograph of the lifting jig. Scale bar is $15 \mathrm{~mm}$. (b) Illustration of the lifting jig with the FDA (c) Initial weight measurement of the lifting jig (d) Measurement result after the lifting test 
(a)

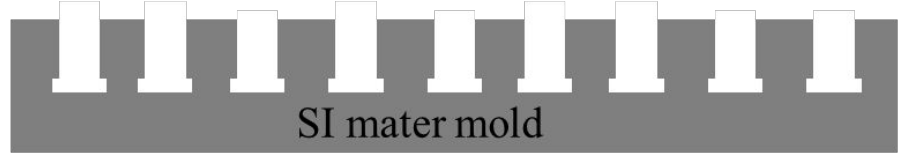

Pour the PDMS $\downarrow$ Degassing

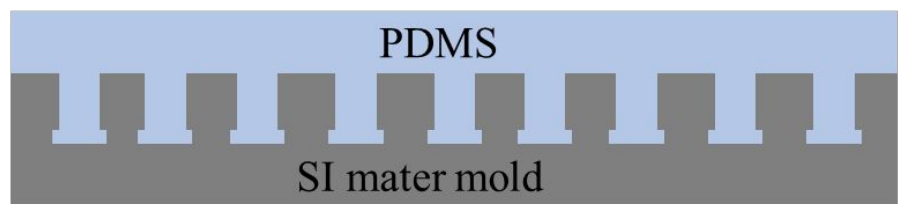

Cure at oven $\downarrow$ Demolding

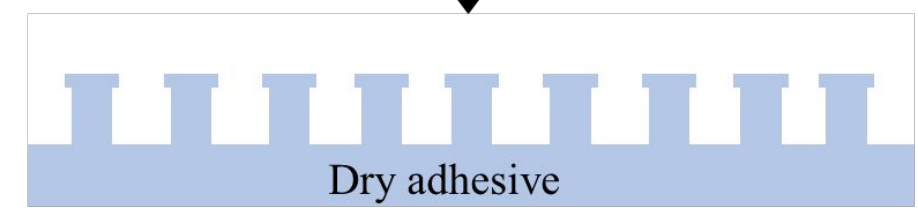

(b)

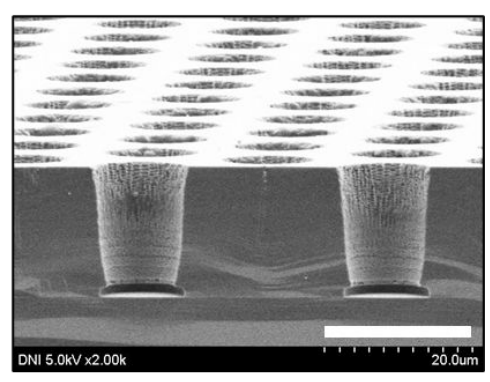

(c)

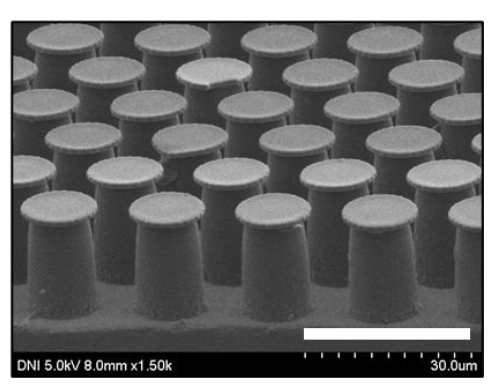

Figure S3. (a) Schematic image of the fabrication process of the micro-structured dry adhesive composed with PDMS. (b) SEM image of the master mold of the dry adhesive. Scale bar is $20 \mu \mathrm{m}$. (c) SEM image of the micro-structured dry adhesive. Dimensions of height and diameter of unit pillar are $20 \mu \mathrm{m}$ and $10 \mu \mathrm{m}$. Scale bar is $30 \mu \mathrm{m}$. 
(a)

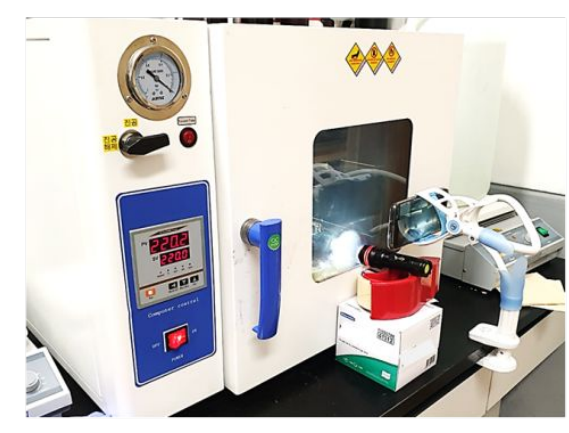

(c)
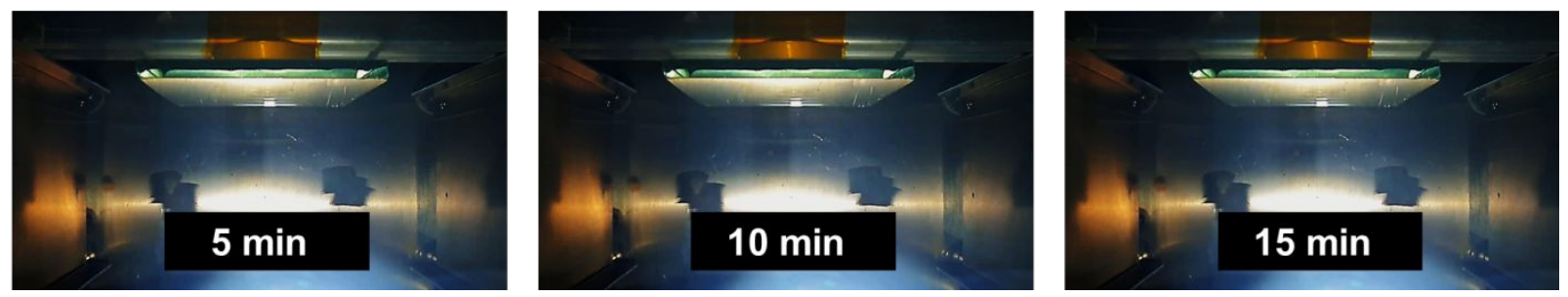

Figure S4. (a) Photograph of experimental setup for adhesion maintaining time in the high temperature (b) Illustration of the experimental method (c) Time-laps images of the adhesion with glass substrate from $5 \mathrm{~min}$ to $15 \mathrm{~min}$ at in $220{ }^{\circ} \mathrm{C}$ 
Table S1. Materials information to obtain the surface energy of the FDA. $\gamma$ is the surface energy, and the subscripts $d$ and $p$ are the dispersion and polar terms, respectively.

\begin{tabular}{ccc}
\hline Material & $\gamma^{d}\left[\mathrm{~mJ} \mathrm{~m}^{-2}\right]$ & $\gamma^{p}\left[\mathrm{~mJ} \mathrm{~m}^{-2}\right]$ \\
\hline Water & 21.8 & 51.0 \\
Glycerol & 33.6 & 30.1 \\
\hline
\end{tabular}

Table S2. Surface energy of various material to calculating the work of adhesion. $\gamma$ is the surface energy, and the subscripts $d$ and $p$ are the dispersion and polar terms, respectively.

\begin{tabular}{ccc}
\hline Material & $\gamma^{d}\left[\mathrm{~mJ} \mathrm{~m}^{-2}\right]$ & $\gamma^{p}\left[\mathrm{~mJ} \mathrm{~m}^{-2}\right]$ \\
\hline FDA & 0.84 & 12.67 \\
PDMS & 21.70 & 1.10 \\
Glass & 28.20 & 35.60 \\
\hline
\end{tabular}




\section{Calculation section}

To analysis a surface energy of the FDA, water and glycerol, well-known material for the surface energy, were used. As shown as Figure S1, the contact angles of water and glycerol were measured as $100.6^{\circ}$ and $102.7^{\circ}$, respectively. Also, the surface energy could be calculated by the Fowkes Model and it could be expressed as follows,

$$
\gamma_{l}(1+\cos \theta)=2\left(\gamma_{l}^{d} \gamma_{s}^{d}\right)^{1 / 2}+2\left(\gamma_{l}^{p} \gamma_{s}^{p}\right)^{1 / 2}
$$

where $\gamma_{l}, \gamma_{s}$ are the surface energy of liquid and solid. Also, $\theta$ is contact angle and superscripts $d$ and $p$ are the dispersion and polar terms, respectively. From a literature, the surface energy of water and glycerol was shown in Table S1. By using these values, the surface energy of the FDA was calculated as $\gamma_{s}^{d}=0.84 \mathrm{~mJ} \mathrm{~m}^{-2}$ and $\gamma_{s}^{p}=12.67 \mathrm{~mJ} \mathrm{~m}^{-2}$, respectively.

The 'work of adhesion' which is the energy required for detachment between two contact surfaces $i$ and $j$. The formula for the work of adhesion between two contact surfaces is expressed as follows,

$$
W_{i j}=\frac{4 \gamma_{i}^{d} \gamma_{j}^{d}}{\gamma_{i}^{d}+\gamma_{j}^{d}}+\frac{4 \gamma_{i}^{p} \gamma_{j}^{p}}{\gamma_{i}^{p}+\gamma_{j}^{p}}
$$

where, $\gamma$ are the surface energy and superscripts $d$ and $p$ are the dispersion and polar terms, respectively. By using surface energy of the FDA and the glass (Table S2), the work of adhesion between the FDA and the glass was calculated as $W_{F D A / g l a s s}=41.02 \mathrm{~mJ} \mathrm{~m}^{-2}$. Also, the work of adhesion between the PDMS and the glass was calculated as $W_{P D M S / \text { glass }}=47.84 \mathrm{~mJ} \mathrm{~m}^{-2}$. The PDMS is well-known material for good adhesion force with the glass and compared with these results, the work of adhesion of the FDA is sufficiently high to exhibit good adhesion with the glass substrate. 


\section{Supporting videos}

Supporting video 1. The stable pull-off strength of FDA under various motions such as lifting, rotation.

Supporting video 2. The first application of FDA for the glass transportation system with the 6 DOF robot arm

Supporting video 3. The second application of FDA for the effective detachment of the glass substrate by using custom-built equipment. 